\title{
Modelling T4 cell count as a marker of HIV progression in the absence of any defense mechanism
}

\author{
Venkata S Sarma Yadavalli* $\quad$ Moremi Morire OreOluwapo Labeodan ${ }^{\dagger}$ \\ Swaminathan Udayabaskaran ${ }^{\ddagger} \quad$ N Forche S $^{\S}$
}

Received: 27 January 2010; Revised: 27 August 2010; Accepted: 8 November 2010

\begin{abstract}
The T4 cell count, which is considered one of the markers of disease progression in an HIV infected individual, is modelled in this paper. The World Health Organisation has recently advocated that countries encourage HIV infected individuals to commence antiretroviral treatments once their T4 cell count drops below 350 cells per $\mathrm{ml}$ of blood (this threshold was formerly 200 cells per $\mathrm{ml}$ of blood). This recommendation is made because when the $\mathrm{T} 4$ cell count is low, the T4 cells are unable to mount an effective immune response against antigens and any such foreign matters in the body, and consequently the individual becomes susceptible to opportunistic infections and lymphomas. A stochastic catastrophe model is developed in this paper to obtain the mean, variance and covariance of the uninfected, infected and lysed T4 cells. The amount of toxin produced in an HIV infected person from the time of infection to a later time may also be obtained from the model. Numerical illustrations of the correlation structures between uninfected and infected T4 cells, and between the infected and lysed T4 cells are also presented.
\end{abstract}

Key words: Toxin, uninfected T4 cells, infected T4 cells, lysed T4 cells, catastrophe stochastic model.

\section{Introduction}

T4 cells, which originate in the bone marrow and mature in the thymus gland, play a dominant role in the immune system of the human body. In fact, these cells amplify immune responses through the release of various cytokine mediators. It has been observed in HIV infected individuals that as a consequence of HIV infection, selective depletion

\footnotetext{
${ }^{*}$ Corresponding author: Department of Industrial and Systems Engineering, University of Pretoria, Pretoria, South Africa, email: yadavalli@postino.up.ac.za

${ }^{\dagger}$ Department of Statistics, University of Pretoria, Pretoria, South Africa.

${ }^{\ddagger}$ Department of Mathematics, and Department of Computer Applications, Vel Tech Dr. RR \& Dr. SR Technical University, Avadi, Chennai, India.

${ }^{\S}$ Department of Statistics, University of Botswana, Gaborone, Botswana.
} 
of T4 cells occurs. When the T4 cell count in such an individual drops, these cells are unable to mount an effective immune response and consequently, the individual becomes susceptible to opportunistic infections and lymphomas. Accordingly, the T4 cell count may be considered a marker of disease progression in an infected individual and the loss of T4 cells accounts for a major part of the immunosuppressive effect of HIV (see, for example, Stein et al. [11], Phillips et al. [8], Feinberg [3] and Sabin et al. [10]).

In the recent past, several researchers have developed various stochastic and deterministic models to describe the temporal progression of the T4 cell count in an HIV infected individual and its relationship to the survival time of the individual. For example, Longini et al. [5] modelled the decline of $\mathrm{T} 4$ cells in HIV infected individuals by means of a continuous-time Markov process in which the state space consists of seven states. These states are the end points of six progression T4 cell count intervals and the beginning of the first interval corresponds to the time of HIV infection and the end of the last interval synchronizes with the time of AIDS diagnosis.

Perelson et al. [7] developed a model for the interaction of HIV with T4 cells by considering four populations, namely uninfected T4 cells, latently infected T4 cells, actively infected T4 cells, and free HIV. Using their model, they examined several features of HIV infection and in particular the process of T4 cell depletion.

De Gruttola and $\mathrm{Tu}[2]$ proposed a model for studying the progression of the T4 cell count and the relationship between different features of this progression and survival time. In their model, they observed the T4 cell count only at certain fixed time points and, using random effects, estimated the T4 trajectory.

Philips et al. [8] developed an extrapolation model based on T4 cell counts measured at discrete points and, using the model, estimated the probability of remaining free of AIDS for up to 25 years after infection with HIV. Cozzi Lepri et al. [1] used multilevel modelling techniques to assess the rate of T4 cell decline in HIV infected individuals and predicted that the rate of T4 cell decline is actually slower at the later stage of the disease.

In the work of Wick [13], the T4 cell loss in an HIV infected individual has been analysed by proposing a model in which the rates of proliferation and programmed cell death (apoptosis) control the rise and fall of the T4 cell count.

In all these works, the stochastic mechanism of HIV production has not been given its due importance in understanding the decline of the T4 cell count and the status of HIV progression in infected individuals. Furthermore, no work appears to be available in literature incorporating the correlation structure between uninfected and infected $\mathrm{T} 4$ cell populations. Also, in HIV related models, there appears to be no work which quantifies the amount of toxins produced during the progression of HIV in infected individuals and its correlation with the loss of T4 cells. In this paper, an attempt is made to fill this gap by building a more realistic stochastic model of HIV production/progression leading to the decline of the T4 cell count in an infected individual.

The organization of this paper is as follows: In Section 2, we develop a catastrophe model of HIV production. The probability generating function for $X(t)$, the number of uninfected cells, $Y(t)$, the number of infected cells at any time $t$ and $Z(t)$, the number of lysed cells up to time $t$ is obtained in Section 3. The means and variances of $X(t), Y(t)$, and $Z(t)$ 
are explicitly found in Section 4. We also obtain explicit expressions for the co-variances between $X(t)$ and $Y(t), Y(t)$ and $Z(t)$, and $Z(t)$ and $X(t)$ in section 4 . The total amount of toxins produced up to time $t$ since the time of HIV infection is quantified and analysed in Section 5. In section 6, a numerical illustration is provided to drive home a satisfactory picture of what happens during the progression of HIV in an infected individual up to the onset of AIDS.

\section{A catastrophe model of infection}

Assume that at time $t=0$, a cell population has $N$ uninfected T4 cells and that an HIV infects one of these cells during the interval $(0, \Delta)$, as $\Delta \rightarrow 0$. The infected cell either splits into two infected cells or undergoes a lysis (bursting of the cell wall due to virus multiplication inside the cell) releasing a random number, $K$, of HIVs which instantaneously infect an equal number of uninfected T4 cells, and the process continues. Furthermore, an independent Poisson arrival of uninfected T4 cells with rate $\alpha$ is assumed into the population of $\mathrm{T} 4$ cells. The process of splitting of an infected cell into two infected cells may be viewed as a birth of an infected cell with the parent surviving, while the event of a lysis of an infected cell may be considered as the death of an infected cell. The death of an infected cell is considered a disaster to the population of uninfected cells. This observation enables us to make the assumption that the population of infected cells undergoes a linear birth and death process, with $\lambda$ and $\mu$ as the birth and death rates, respectively, and that the population of uninfected cells is subject to disasters occurring at the event of the death of an infected cell.

Let $X(t)$ and $Y(t)$ denote respectively the number of uninfected and infected cells at time $t$. Then, by the initial condition, we have $X(0+)=N-1$ and $Y(0+)=1$, where $N$ is sufficiently large and fixed. Let $Z(t)$ represent the number of cells that have undergone lysis up to time $t$. Since there is an independent arrival of T4 cells into the population of T4 cells, it is easy to see that

$$
X(t)+Y(t)+Z(t) \geq N
$$

We assume that $K$ has a discrete distribution defined by

$$
\operatorname{Pr}(K=r)=\pi_{r}, \quad r=0,1,2, \ldots
$$

Let $h(s)$ be the probability generating function of $K$ defined by $h(s)=E\left[s^{K}\right]=\sum_{r=0}^{\infty} \pi_{r} s^{r}$. The vector process $(X(t), Y(t), Z(t))$ is clearly Markov and we proceed to obtain its probability generating function in the next section.

\section{The probability generating function}

We define the probability generating function of $(X(t), Y(t), Z(t))$,

$$
G(u, v, w ; t)=E\left[u^{X(t)} v^{Y(t)} w^{Z(t)}\right] .
$$


Then it is easy to see that $G(u, v, w ; 0)=u^{N-1} v$. To derive an expression for $G(u, v, w ; t)$, we first define the probability function

$$
p(i, j, k ; t)=\operatorname{Pr}\{X(t)=i, Y(t)=j, Z(t)=k\} .
$$

Then, using the laws of probability theory, we obtain

$$
\begin{aligned}
\frac{\partial p(i, j, k: t)}{\partial t}= & -\{j(\lambda+\mu)+\alpha\} p(i, j, k ; t)+\alpha p(i-1, j, k ; t)+(j-1) \lambda p(i, j-1, k ; t) \\
& +\sum_{r=0}^{j+1}(j+1-r) \mu p(i+r, j+1-r, k-1 ; t) \pi_{r}
\end{aligned}
$$

From (1), following Bailey (1975), it may be shown that the probability generating function $G(u, v, w ; t)$ satisfies the partial differential equation

$$
\frac{\partial G}{\partial t}=-(\lambda+\mu) v \frac{\partial G}{\partial v}-\alpha(1-u) G+\lambda v^{2} \frac{\partial G}{\partial v}+\mu w \sum_{r=0}^{\infty} \pi_{r} u^{-r} v^{r} \frac{\partial G}{\partial v},
$$

with the initial condition $G(u, v, w ; 0)=u^{N-1} v$. After simplification, (2) becomes

$$
\frac{\partial G}{\partial t}=-\alpha(1-u) G+\left\{-(\lambda+\mu) v+\lambda v^{2}+\mu w h\left(\frac{v}{u}\right)\right\} \frac{\partial G}{\partial v} .
$$

Equation (3) is not easily solvable, even for any simple form of the generating function $h(\cdot)$. However, we can obtain from (3) the various moments of $X(t), Y(t)$ and $Z(t)$. Accordingly, in the next section, we study the moment structure of the process $(X(t), Y(t), Z(t))$. We also study the covariance structure of $X(t), Y(t)$ and $Z(t)$.

\section{The moment structure of $(X(t), Y(t), Z(t))$}

Upon adopting the notation

$$
\begin{array}{llll}
M_{X}(t)=E[X(t)], & M_{X}^{(2)}(t)=E[X(t)\{X(t)-1\}], & & M_{X Y}(t)=E[X(t) Y(t)], \\
M_{Y}(t)=E[Y(t)], & M_{Y}^{(2)}(t)=E[Y(t)\{Y(t)-1\}], & & M_{Y Z}(t)=E[Y(t) Z(t)], \\
M_{Z}(t)=E[Z(t)], & M_{Z}^{(2)}(t)=E[Z(t)\{Z(t)-1\}], & & M_{Z X}(t)=E[Z(t) X(t)],
\end{array}
$$

the system of equations

$$
\begin{aligned}
& \frac{\partial M_{X}(t)}{\partial t}=\alpha-\mu h^{\prime}(1) M_{Y}(t) \\
& \frac{\partial M_{Y}(t)}{\partial t}=\alpha M_{Y}(t) \\
& \frac{\partial M_{Z}(t)}{\partial t}=\mu M_{Y}(t)
\end{aligned}
$$


T4 cell count as a marker of HIV progression in the absence of any defense mechanism 117

$$
\begin{aligned}
& \frac{\partial M_{X}^{(2)}(t)}{\partial t}=-2 \mu h(1) M_{X Y}(t)+2 \alpha M_{X}(t)+d M_{Y}(t) \\
& \frac{\partial M_{Y}^{(2)}(t)}{\partial t}=2 a M_{Y}^{(2)}(t)+c M_{Y}(t) \\
& \frac{\partial M_{Z}^{(2)}(t)}{\partial t}=2 \mu M_{Y Z}(t) \\
& \frac{\partial M_{X Y}(t)}{\partial t}=a M_{X Y}(t)+b M_{Y}(t)-\mu h^{\prime}(1) M_{Y}^{(2)}(t) \\
& \frac{\partial M_{Y Z}(t)}{\partial t}=a M_{Y Z}(t)+\mu M_{Y}^{(2)}(t)-\mu h^{\prime}(1) M_{Y}(t) \\
& \frac{\partial M_{Z X}(t)}{\partial t}=a M_{Z}(t)+\mu M_{X Y}(t)-\mu h^{\prime}(1) M_{Y}(t)-\mu h^{\prime}(1) M_{Y Z}(t)
\end{aligned}
$$

emerges, where

$$
\begin{array}{ll}
a=\lambda-\mu+\mu h^{\prime}(1), & c=2 \lambda+\mu h^{\prime \prime}(1), \\
b=\alpha-\mu h^{\prime}(1)-\mu h^{\prime \prime}(1), & d=2 \mu h^{\prime}(1)+\mu h^{\prime \prime}(1) .
\end{array}
$$

Noting that

$$
\begin{array}{lll}
M_{X}(0)=N-1, & M_{X}^{(2)}(0)=(N-1)(N-2), & M_{X Y}(0)=N-1, \\
M_{Y}(0)=1, & M_{Y}^{(2)}(0)=0, & \\
M_{Y Z}(0)=0, & M_{Z}^{(2)}(0)=0, & M_{Z X}(0)=0,
\end{array}
$$

and taking Laplace transforms in (4)-(12), yields

$$
\begin{aligned}
M_{X}^{*}(s)= & \frac{N-1}{s}+\frac{\alpha}{s^{2}}-\frac{\mu h^{\prime}(1)}{s(s-a)}, \\
M_{Y}^{*}(s)= & \frac{1}{s-a}, \\
M_{Z}^{*}(s)= & \frac{\mu}{s(s-a)}, \\
M_{X}^{(2) *}(s)= & \frac{(N-1)(N-2)}{s}+\frac{d}{s(s-a)}-2 \mu h^{\prime}(1)\left\{\frac{N-1}{s(s-a)}+\frac{b(s-2 a)-\mu h^{\prime}(1) c}{s(s-a)^{2}(s-2 a)}\right\} \\
& +2 \alpha\left\{\frac{N-1}{s^{2}}+\frac{\alpha}{s^{3}}-\frac{\mu h^{\prime}(1)}{s^{2}(s-a)}\right\}, \\
M_{Y}^{(2) *}(s)= & \frac{c}{(s-a)(s-2 a)}, \\
M_{Z}^{(2) *}(s)= & 2 \mu^{2}\left\{\frac{c+h^{\prime}(1)(s-2 a)}{s(s-a)^{2}(s-2 a)}\right\} \\
M_{X Y}^{*}(s)= & \frac{N-1}{s-a}+\frac{b(s-2 a)-\mu h^{\prime}(1) c}{(s-a)^{2}(s-2 a)},
\end{aligned}
$$




$$
\begin{aligned}
& M_{Y Z}^{*}(s)=\mu\left\{\frac{c+h^{\prime}(1)(s-2 a)}{(s-a)^{2}(s-2 a)}\right\} \text { and } \\
& M_{Z X}^{*}(s)=\mu\left\{\frac{N-1}{s(s-a)} \frac{h^{\prime}(1)}{s(s-a)}+\frac{b-\mu\left(h^{\prime}(1)\right)^{2}(s-2 a)-2 \mu h^{\prime}(1) c}{s(s-a)^{2}(s-2 a)}+\frac{\alpha}{s^{2}(s-a)}\right\} .
\end{aligned}
$$

Inverting these equations, we obtain

$$
\begin{aligned}
M_{X}(t)= & N-1+\alpha t-\frac{\mu h^{\prime}(t)}{a}\left(e^{a t}-1\right), \\
M_{Y}(t)= & e^{a t} \\
M_{Z}(t)= & \frac{\mu}{a}\left(e^{a t}-1\right), \\
M_{X}^{(2)}(t)= & (N-1)(N-2) \frac{d}{a}\left(e^{a t}-1\right) \\
& +\frac{\alpha}{a^{2}}\left\{2(N-1) a^{2} t+\alpha(a t)^{2}-2 \mu h^{\prime}(1)\left(e^{a t}-a t e^{a t}-1\right)\right\} \\
& -2 \mu h^{\prime}(1)\left\{\frac{N-1}{a}\left(e^{a t}-1\right)-\frac{\mu h^{\prime}(1) c}{2 a^{2}\left(e^{2 a t}-2 a t e^{a t}-1\right)}-\frac{b}{a^{2}}\left(e^{a t}-a t e^{a t}-1\right)\right\}, \\
M_{Y}^{(2)}(t)= & \frac{c}{a}\left(e^{2 a t}-e^{a t}\right), \\
M_{Z}^{(2)}(t)= & \frac{\mu^{2}}{a^{2}}\left\{\frac{c}{a}\left(e^{2 a t}-2 a t e^{a t}-1\right)-2 h^{\prime}(1)\left(e^{a t}-a t e^{a t}-1\right)\right\} \\
M_{X Y}(t)= & (N-1+b t) e^{a t}-\frac{\mu h^{\prime}(1) c}{a^{2}}\left(e^{2 a t}-a t e^{a t}-e^{a t}\right) \\
M_{Y Z}(t)= & \mu\left\{\frac{c}{a^{2}}\left(e^{2 a t}-a t e^{a t}-e^{a t}\right)+h^{\prime}(1) t e^{a t}\right\} \\
M_{Z X}(t)= & \frac{\mu}{a}\left\{\left(N-1-h^{\prime}(1)\right)\left(e^{a t}-1\right)-\frac{(b-\mu)\left(h^{\prime}(1)^{2}\right)}{a}\left(e^{a t}-a t e^{a t}-1\right)\right\} \\
+ & \frac{\mu}{a}\left\{\frac{\alpha}{a}\left(e^{a t}-a t-1\right)-\frac{\mu h^{\prime}(1) c}{a^{2}}\left(e^{2 a t}-2 a t e^{a t}-1\right)\right\}
\end{aligned}
$$

\section{The amount of toxin produced}

Whenever an infected cell appears, a quantity of toxic substance is produced in the blood. The estimation of the total amount of toxins produced by the infected cells since the beginning of the HIV infection up to any time is useful in knowing the level of HIV infection. In this section, we quantify the total amount of the toxins and obtain its mean and variance. We assume that the amount of toxins produced at time $t$ is proportional to the number of infected cells present at time $t$. The total amount of toxins produced up to time $t$ since the beginning of the HIV infection is given by the stochastic integral

$$
W(t)=\int_{0}^{t} Y(u) \mathrm{d} u .
$$

The integral in (22) exists almost surely and has been studied very extensively in several biological applications by several researchers (see, for example, Puri [9], Jagers [4], Pakes [6], and Udayabaskaran and Sudalaiyandi [12]). 
T4 cell count as a marker of HIV progression in the absence of any defense mechanism 119

We proceed to obtain the joint moment generating function of $Y(t)$ and $W(t)$ defined by

$$
H(u, v ; t)=E\left[u^{Y(t)} e^{-v W(t)} \mid Y(0)=1\right] .
$$

Fixing the occurrence of the first event since time $t=0$ and using probabilistic arguments, we obtain the integral equation

$$
\begin{aligned}
H(u, v ; t)= & u e^{-(\lambda+\mu+\varepsilon) \tau}+\lambda \int_{0}^{t} e^{-(\lambda+\mu+\varepsilon) \tau}\left\{H(u, v ; t-\tau\}^{2} \mathrm{~d} \tau\right. \\
& +\mu \int_{0}^{t} e^{-(\lambda+\mu+\varepsilon) \tau} h(H(u, v ; t-\tau)) \mathrm{d} \tau
\end{aligned}
$$

where

$$
h(s)=\sum_{r=0}^{\infty} \pi_{r} s^{r}
$$

is the generating function of the number of HIV's produced at the time of a lysis. From (32) we are able to obtain the mean and variance of $W(t)$ and the correlation structure of $W(t)$ with $Y(t)$.

Differentiating (32) with respect to $v$ at $(u=1, v=0)$, we get

$$
\begin{aligned}
M_{W}(t)= & t e^{-(\lambda+\mu) t}+\lambda \int_{0}^{t} e^{-(\lambda+\mu) \tau}\left[2 M_{W}(t-\tau)+\tau\right] \mathrm{d} \tau \\
& +\mu \int_{0}^{t} e^{-(\lambda+\mu) \tau}\left[h^{\prime}(1) M_{W}(t-\tau)+\tau\right] \mathrm{d} \tau .
\end{aligned}
$$

Differentiating (32) twice with respect to $v$ at $(u=1, v=0)$, we get

$$
\begin{aligned}
M_{W W}(t)= & t^{2} e^{-(\lambda+\mu) t}+(\lambda+\mu) \int_{0}^{t} e^{-(\lambda+\mu) \tau} \tau^{2} \mathrm{~d} \tau \\
& +\left[2 \lambda+\mu h^{\prime}(1)\right] \int_{0}^{t} e^{-(\lambda+\mu) \tau} M_{W W}(t-\tau) \mathrm{d} \tau \\
& +\left[2 \lambda+\mu h^{\prime \prime}(1)\right] \int_{0}^{t} e^{-(\lambda+\mu) \tau}\left\{M_{W}(t-\tau)\right\}^{2} \mathrm{~d} \tau \\
& +2\left[2 \lambda+\mu h^{\prime}(1)\right] \int_{0}^{t} e^{-(\lambda+\mu) \tau} \tau M_{W}(t-\tau) \mathrm{d} \tau
\end{aligned}
$$

Differentiating (32) with respect to $u$ and $v$ at $(u=1, v=0)$, we get

$$
\begin{aligned}
M_{Y W}(t)= & t e^{-(\lambda+\mu) t}+\left[2 \lambda+\mu h^{\prime}(1)\right] \int_{0}^{t} e^{-(\lambda+\mu) \tau} M_{Y W}(t-\tau) \mathrm{d} \tau \\
& +\left[2 \lambda+\mu h^{\prime \prime}(1)\right] \int_{0}^{t} e^{-(\lambda+\mu) \tau} M_{Y}(t-\tau) M_{W}(t-\tau) \mathrm{d} \tau \\
& +\left[2 \lambda+\mu h^{\prime}(1)\right] \int_{0}^{t} e^{-(\lambda+\mu) \tau} \tau M_{Y}(t-\tau) \mathrm{d} \tau .
\end{aligned}
$$


On applying Laplace transforms to the expressions in (33), (34) and (35) it follows that

$$
\begin{aligned}
M_{W}^{*}(s)= & \frac{1}{s(s-a)}, \\
M_{W W}^{*}(s)= & \frac{2}{s(s-a)(s+\lambda+\mu)}+\frac{2 \lambda+\mu h^{\prime \prime}(1)}{a^{2}}\left\{\frac{1}{(s-2 a)}-\frac{2 a}{(s-a)^{2}}+\frac{1}{s}\right\} \\
& +\frac{2\left[2 \lambda+\mu h^{\prime}(1)\right]}{a^{2}}\left\{\frac{a}{(s-a)^{2}}-\frac{1}{s-a}+\frac{1}{s}\right\}, \\
M_{Y W}^{*}(s)= & \frac{1}{(s-a)^{2}}+\frac{2 \lambda+\mu h^{\prime \prime}(1)}{a^{2}} \frac{1}{(s-2 a)}-\frac{a}{(s-a)^{2}}+\frac{1}{s-a} .
\end{aligned}
$$

On inversion, these equations yield

$$
\begin{aligned}
M_{W}(t)= & \frac{1}{a}\left(e^{a t}-1\right), \\
M_{W W}(t)= & 2 a\left\{\frac{1}{a(\lambda+\mu+a)} e^{a t}+\frac{1}{(\lambda+\mu)(\lambda+\mu+a)} e^{-(\lambda+\mu) t}-\frac{1}{a(\lambda+\mu)}\right\} \\
& +\frac{2 \lambda+\mu h^{\prime \prime}(1)}{a^{3}}\left(e^{2 a t}-2 a t e^{a t}-1\right)-\frac{2\left[2 \lambda+\mu h^{\prime}(1)\right]}{a^{2}}\left(e^{a t}-a t e^{a t}-1\right), \\
M_{Y W}(t)= & t e^{a t}+\frac{2 \lambda+\mu h^{\prime \prime}(1)}{a^{2}}\left(e^{2 a t}-a t e^{a t}-e^{a t}\right) .
\end{aligned}
$$

\section{$6 \quad$ Numerical illustration}

The behaviour of the means of $X(t), Y(t)$ and $Z(t)$ and the correlation coefficient between $X(t)$ and $Y(t)$ (i.e. $R_{X Y}$ ) and that between $Y(t)$ and $Z(t)$ (i.e. $R_{Y Z}$ ) are studied as functions of time by means of numerical examples in this section. For this purpose, we assume arbitrary values of $\alpha=100.0, \lambda=0.20, \mu=0.10$, and vary $t$ from 0.5 to 0.8 in steps of 0.5. The results are highlighted in Tables 1 to 4 .

\begin{tabular}{cccc}
\hline$t$ & $E[X(t)]$ & $E[Y(t)]$ & $E[Z(t)]$ \\
\hline 0.50 & 10.0483 & 0.0017 & 0.0007 \\
1.00 & 10.0972 & 0.0030 & 0.0018 \\
1.50 & 10.1452 & 0.0052 & 0.0038 \\
2.00 & 10.1917 & 0.0090 & 0.0073 \\
2.50 & 10.2357 & 0.0156 & 0.0133 \\
3.00 & 10.2753 & 0.0156 & 0.0133 \\
3.50 & 10.2753 & 0.0271 & 0.0237 \\
4.00 & 10.3259 & 0.0815 & 0.0731 \\
4.50 & 10.3216 & 0.1412 & 0.1274 \\
5.00 & 10.2775 & 0.2447 & 0.2215 \\
5.50 & 10.1644 & 0.4241 & 0.3846 \\
6.00 & 9.9316 & 1.2741 & 1.1574 \\
6.50 & 9.4916 & 0.7351 & 0.6674 \\
7.00 & 8.6923 & 2.2083 & 2.0067 \\
7.50 & 7.2702 & 3.8276 & 3.4788 \\
8.00 & 4.7688 & 6.6342 & 6.0302 \\
\hline
\end{tabular}

Table 1: $E[X(t)], E[Y(t)]$ and $E[Z(t)]$ versus $t$ for $\alpha=100.0, \lambda=0.20, \mu=0.10$. 


\begin{tabular}{ccc}
\hline$t$ & $R_{X Y}$ & $R_{Y Z}$ \\
\hline 0.50 & -0.8770 & 0.8406 \\
1.00 & -0.9226 & 0.9130 \\
1.50 & -0.9616 & 0.9446 \\
2.00 & -0.9829 & 0.9641 \\
2.50 & -0.9934 & 0.9769 \\
3.00 & -0.9970 & 0.9854 \\
3.50 & -0.9989 & 0.9909 \\
4.00 & -0.9995 & 0.9944 \\
4.50 & -0.9998 & 0.9966 \\
5.00 & -0.9999 & 0.9980 \\
5.50 & -1.0000 & 0.9988 \\
6.00 & -1.0000 & 0.9993 \\
6.50 & -1.0000 & 0.9996 \\
7.00 & -1.0000 & 0.9998 \\
7.50 & -1.0000 & 0.9999 \\
8.00 & -1.0000 & 0.9999 \\
\hline
\end{tabular}

Table 2: $\quad R_{X Y}$ and $R_{Y Z}$ versus $t$ for $\alpha=100.0, \lambda=0.20, \mu=0.10$.

From the numerical results it may be seen that the number of uninfected T4 cells present at any instant of time decreases (Table 1) and that of the infected cells (Table 2) increases with time, as expected. This implies that the mean of the cumulative quantity of toxin produced should also increase with time and Table 1 confirms this result. It is also observed that the correlation between $X(t)$ and $Y(t)$ remains negative (Table 2), whereas the correlation between $Y(t)$ and $Z(t)$ is positive throughout the period under consideration (Table 2).

Furthermore, as the rate of arrival of uninfected T4 cells increases $(\alpha=100)$, the mean number of uninfected T4 cells present at time instant 0.5 increases. However, the means of the number of infected cells and that of the cumulative quantity of toxin produced remain the same irrespective of the values of $\alpha$ (Table 3). Also, there is a negative correlation between $X(t)$ and $Y(t)$ (Table 4). Correlation between $Y(t)$ and $Z(t)$ exists, but nothing can be said about the nature of its variation (Table 4) with respect to $\alpha$.

\begin{tabular}{cccc}
\hline$\alpha$ & $E[X(t)]$ & $E[Y(t)]$ & $E[Z(t)]$ \\
\hline 100.00 & 10.0483 & 0.0017 & 0.0007 \\
200.00 & 10.0983 & 0.0017 & 0.0007 \\
300.00 & 10.1483 & 0.0017 & 0.0007 \\
400.00 & 10.1983 & 0.0017 & 0.0007 \\
500.00 & 10.2483 & 0.0017 & 0.0007 \\
600.00 & 10.2983 & 0.0017 & 0.0007 \\
700.00 & 10.3483 & 0.0017 & 0.0007 \\
800.00 & 10.3983 & 0.0017 & 0.0007 \\
900.00 & 10.4483 & 0.0017 & 0.0007 \\
1000.00 & 10.4983 & 0.0017 & 0.0007 \\
\hline
\end{tabular}

Table 3: $E[X(t)], E[Y(t)]$ and $E[Z(t)]$ versus $\alpha$ for $t=0.50, \lambda=0.20, \mu=0.01$. 


\section{Conclusion}

In this paper, we have obtained the mean number of uninfected, infected and lysed T4 cells in an HIV infected individual. Unlike other models proposed (see, for example, Longini et al. [5], Perelson et al. [7], De Gruttola and Tu [2], Philips et al. [8], Cozzi-Lepri et al. [1] and Wick [13]), our model not only provides the moment structure of the variables, but also the co-variance relationship between them. Hence we have been able to build on previous models establishing the T4 cell count as a marker of the disease progression. Also we were able to model the quantity of toxin produced as a function of time in an HIV infected individual.

\begin{tabular}{ccc}
\hline$\alpha$ & $R_{X Y}$ & $R_{Y Z}$ \\
\hline 100.00 & -0.8770 & 0.8406 \\
200.00 & -0.7595 & 0.8406 \\
300.00 & -0.6578 & 0.8406 \\
400.00 & -0.6036 & 0.8406 \\
500.00 & -0.5674 & 0.8406 \\
600.00 & -0.5262 & 0.8406 \\
700.00 & -0.4929 & 0.8406 \\
800.00 & -0.4688 & 0.8406 \\
900.00 & -0.4479 & 0.8406 \\
1000.00 & -0.4297 & 0.8406 \\
\hline
\end{tabular}

Table 4: $\quad R_{X Y}$ and $R_{Y Z}$ versus $\alpha$ for $t=0.50, \lambda=0.20, \mu=0.01$.

\section{References}

[1] Cozzi-Lepri A, Sabin CA, Pezzoti PD, Philips AN \& Rezza G, 1997, Is there a general tendency for the CD4 lymphocyte decline to speed up during human immunodeficiency virus infection? Evidence from the Italian seroconversion study, Journal of Infectious Diseases, 175, pp. 775-780.

[2] De Gruttola V \& TU XM, 1994, Modeling progression of CD4-lymphocyte count and the relationship to survival time, Biometrics, 50, pp. 1003-1014.

[3] Feinberg MB, 1996, Changing the natural history of HIV disease, Lancet, 348, pp. 239-246.

[4] JAGERS P, 1967, Integrals of branching processes, Biometrika, 54, pp. 263-271.

[5] Longini LM, Clark WS, Gardener LI \& Brundage J, 1991, The dynamic of CD 4+T-lymphocyte decline in HIV-infected individuals - A Markov modelling approach, Journal of Acquired Immune Deficiency Syndromes, 4, pp. 1141-1147.

[6] Pakes AG, 1975, On Markov branching processes with immigration, The Indian Journal of Statistics, 37(1), pp. 129-138.

[7] Perelson AS, Kirschner DE \& De Boer R, 1993, The dynamics of HIV infection of CD4 $+T$ cells, Mathematical Biosciences, 114, pp. 81-125.

[8] Philips AN, Pezzoti P, CozziI-Lepri A \& Rezza G, 1994, CD4 lymphocyte count as a determinant of the time from HIV seroconversion to AIDS and death from AIDS: Evidence from the Italian seroconversion study, AIDS, 8, pp. 1299-1305.

[9] PURI PS, 1966, On the homogeneous birth-and-death process and its integral, Biometrika, 53, pp. $61-71$.

[10] Sabin CA, Mocroft A \& LePri AC, 1998, Cofactors and markers of disease progression in human immunodeficiency virus infection, Journal of the Royal Statistical Society, 161(2), pp. 177-189. 
T4 cell count as a marker of HIV progression in the absence of any defense mechanism 123

[11] Stein DS, Konvick JA \& Vermund SH, 1992, CD4 + lymphocyte cell enumeration for prediction of a clinical course of human immunodeficiency virus disease: A review, Journal of Infectious Diseases, 165, pp. 352-363.

[12] Udayabaskaran S \& Sudalaiyandi G, 1986, On a stochastic integral of a branching process, Journal of Mathematical Biology, 24, pp. 467-472.

[13] Wick D, 1999, On T-cell dynamics and the hyperactivation theory of AIDS pathogenesis, Mathematical Biosciences, 158, pp. 127-144. 
